\title{
Predictors of 1-Year Treatment Outcome in Bulimia Nervosa
}

\author{
Cynthia M. Bulik, Patrick F. Sullivan, Peter R. Joyce, Frances A. Carter, and Virginia V. McIntosh
}

\begin{abstract}
We examined predictors of outcome 1 year after completion of a randomized clinical trial assessing the additive efficacy of two forms of exposure with response prevention to a core of cognitive-behavioral therapy (CBT) for bulimia nervosa (BN). One hundred one women who met DSM-III-R criteria for BN, and who completed the clinical trial, were available for follow-up at 1 year. Predictor variables were assessed prospectively and partitioned temporally to reflect lifetime history (including personality), pretreatment clinical status, and posttreatment clinical status. Outcome was based on the frequency of binging and purging in the 3 months before assessment based on carefully constructed lifechart interviews. A series of stepwise logistic regressions were performed to determine independent predictors of 1-year outcome while controlling for treatment received. Demographic variables were unrelated to treatment outcome. A history of obesity was predictive of poor outcome, whereas a history of alcohol dependence decreased the odds of
\end{abstract}

$\mathbf{I}^{\mathrm{D}}$ DENTIFICATION OF VARIABLES that predict treatment outcome in women with bulimia nervosa (BN) is critical if we are to increase the degree of sophistication with which we treat the disorder. Understanding predictors of outcome could theoretically facilitate matching treatments to individuals based on their clinical profiles at presentation. Likewise, accurate prediction of clinical course based on status at the end of a circumscribed treatment could assist with treatment planning for individuals who are likely to relapse.

Despite the increasingly large number of studies addressing the outcome of BN, little consensus has been reached regarding predictors of outcome (see Keel and Mitchell ${ }^{1}$ for a review). Across studies, putative predictors of outcome have fallen into approximately seven categories: demography (age, socioeconomic status, marital status, and education); behavioral symptoms of the disorder (severity of binging and purging and duration of illness);

From the Department of Psychiatry and Virginia Institute for Psychiatric and Behavioral Genetics, Virginia Commonwealth University, Richmond, VA; and the University Department of Psychological Medicine, Christchurch School of Medicine, Christchurch, New Zealand.

Address reprint requests to Cynthia $M$. Bulik, Ph.D., Department of Psychiatry, Virginia Commonwealth University, Medical College of Virginia Campus, PO Box 980710, Richmond, VA 23298.

Copyright $\odot 1998$ by W.B. Saunders Company

$0010-440 X / 98 / 3904-0002 \$ 03.00 / 0$ poor outcome. High self-directedness on the Temperament and Character Inventory (TCl) predicted favorable outcome at 1 year, whereas personality disorder symptoms were not predictive. Pretreatment global functioning, bulimia scores on the Eating Disorders Inventory (EDI), and the presence of major depression predicted poor outcome. Posttreatment binging, food restriction, and urges to binge on a cue reactivity assessment predicted poor outcome at 1 year. The character trait of self-directedness is a strong predictor of good outcome for CBT, and methods to enhance this trait may be worthy of investigation. Low global functioning and the presence of major depression at presentation may require additional treatment than focused CBT for BN. Our results argue for treatment goals that include abstinence from binging and restricting and decreases in urges to binge in response to high-risk cues.

Copyright (c) 1998 by W.B. Saunders Company

psychological or cognitive features (body dissatisfaction and drive for thinness); axis I comorbidity; axis II comorbidity; associated personality or psychological characteristics (self-esteem and ineffectiveness); and family history (e.g., depression, alcoholism, and obesity). A replicable set of predictors of outcome has not yet been identified.

Many studies have failed to account for the temporal nature of the predictor variables. Predictors often include items that are assessed at pretreatment about the past time frame (e.g., history of weight fluctuations or lifetime history of depression), at pretreatment about the present time frame (e.g., frequency of pretreatment binging and purging), and at the end of treatment about the present time frame (e.g., frequency of posttreatment binging and purging). Determining the pretreatment characteristics of an individual that predict longterm outcome poses a very different question than examining the predictive capacity of posttreatment status. Examination of pretreatment variables addresses characteristics of the treatment-seeking individual that are predictive of outcome, whereas examination of posttreatment variables addresses characteristics of the individual, characteristics of the treatment, and the individual-by-treatment interaction. The first question is relevant to treatment matching, and the second is more relevant to stepped care and relapse prevention.

The definition of outcome in the literature has 
also varied. Definitions have ranged from "no longer meeting DSM-III-R criteria for BN" to "abstinent" from binging and purging for a specified duration. Each of these definitions poses a unique set of difficulties. For example, an individual no longer meeting criteria for $\mathrm{BN}$ can still be binging and purging regularly. Similarly, given that $\mathrm{BN}$ is often a waxing and waning condition, ${ }^{2}$ abstinence can be precarious if not assessed over a sufficiently long interval. Finally, complicating all assessment for $\mathrm{BN}$ is the uncertain nature of self-report and the absence of objective tests verifying clinical status.

Although the current investigation could not address all of these issues, its primary goal was to examine predictors of outcome from BN 1 year after the completion of cognitive-behavior therapy (CBT) by partitioning predictors temporally into lifetime (including personality), pretreatment, and posttreatment categories.

\section{METHODS}

\section{Participants}

The data from this report are taken from the 1-year follow-up assessment of a randomized clinical trial examining the additive efficacy of two forms of exposure with response prevention to a core of CBT for the treatment of BN. Initial results from the clinical trial have been presented elsewhere. ${ }^{3}$ Inclusion criteria for this study were female gender, age 17 to 45 years, and the presence of a current primary DSM-III-R diagnosis of BN. Exclusion criteria were current anorexia nervosa, current obesity (body mass index $>30 \mathrm{~kg} / \mathrm{m}^{2}$ ), current severe major depression (including serious suicidal intent or inability to engage in cognitive therapy due to severity of depression), current severe medical illness or severe medical complications of $\mathrm{BN}$, or current use of psychoactive medication and unwillingness to undergo a supervised drug wash-out period. Recruitment was broad-based and included direct mailings to general practitioners and mental health providers and advertisements at local universities, polytechnic institutes, and in local media.

\section{Procedure}

This study was approved by the Southern Regional Health Authority (Canterbury) and the University of Canterbury ethical committees. All participants signed written informed consent. Before treatment, participants underwent an extensive psychiatric and behavioral assessment. Data from this assessment are used in this report as the pretreatment predictors of 1-year outcome. The presence of axis I and II disorders were determined with the Structured Clinical Interview for DSM-III-R ([SCID] Patient version and version for Personality Disorders). ${ }^{4.5}$ In addition, the assessing clinician completed a structured clinical interview developed for this study and based on constructs outlined in the Eating Disorders Examination ${ }^{6}$ to determine the frequency of objective binging (eating an unusually large amount of food in a short period of time and feeling out of control), ${ }^{7}$ frequency of purging (episodes of vomiting and laxative use), and intensity and frequency of body dissatisfaction and food restriction over the previous 2 weeks; the Hamilton Depression Rating Scale (HDRS); and the Global Assessment of Functioning Scale (GAFS). The clinician-rated body dissatisfaction and food restriction items were first combined to give an intensity $x$ frequency index and then partitioned into quartiles. Participants also completed the Eating Disorders Inventory (EDI) ${ }^{9}$ (drive for thinness, bulimia, and body dissatisfaction scales are reported herein) and the Temperament and Character Inventory (TCI). ${ }^{10}$

Before beginning treatment, a cue reactivity assessment was performed that examined physiological (heart rate and blood pressure), cognitive (urge to binge and urge to purge), and affective (subjective units of distress [SUDS]) responses to the presentation of individualized high-risk foods under controlled laboratory conditions. The procedure was standardized across all subjects for time of day and time since last meal. Before the assessment, subjects were given a complete description of the procedure, and a cue inventory was performed that identified "high-risk" foods that were most likely to trigger a binge. On the day of the cue reactivity assessment, after 10 minutes of baseline, a platter containing the highest risk food was brought into the room. The participant was asked to view, smell, and finally eat as much of the food as she could. Subjects reported their urge to binge, urge to purge, and SUDS at 2-minute intervals. Physiological and self-report recordings continued throughout the procedure. The food was removed after the subjects chose to discontinue eating (or after they made a firm decision not to eat). Recordings continued for approximately 10 more minutes, after which subjects were appropriately debriefed. The peak measures for the self-report ratings of urge to binge, urge to purge, and SUDS are examined in this report.

\section{Treatment Conditions}

All treatment was manual-based (treatment manuals are available for purchase by contacting the first author), and conducted by trained female clinical psychologists. All individuals who entered the study first received eight sessions of CBT. For the first 2 weeks, sessions were twice weekly, followed by weekly sessions for 4 weeks. CBT consisted of self-monitoring, normalization of meals, psychoeducation, self-monitoring, cue identification, challenging automatic thoughts, thought restructuring, chaining, and relapse prevention. Specific goals for each session were outlined clearly in the manuals, and homework was assigned for each module.

Participants were then randomized to either exposure with response prevention to prebinge cues (B-ERP), exposure with response prevention to prepurge cues (P-ERP), or a control condition of relaxation training. The nature of these treatments is described elsewhere in greater detail. ${ }^{3}$ Briefly, in the B-ERP condition, individuals were exposed to cue combinations that preceded binge eating, did not actually eat any foods, and were prevented from engaging in the binge response. In the P-ERP condition, subjects were exposed to prepurge cue combinations, which included eating high-risk foods, and the purging response was prevented. Relaxation involved guided progressive deepmuscle relaxation exercises. Each of the experimental treatments included eight sessions over 6 weeks. All ERP and relaxation sessions were a minimum of 50 minutes; however, in the ERP conditions, exposure continued until physiological and 
self-report measures approached baseline. Thus, although the number of sessions was equated across experimental treatments, the actual duration of sessions was longer in the ERP conditions than in relaxation treatment.

\section{Posttreatment Assessment and 6-Month Follow-up Status}

Following the last treatment session, all participants underwent a final posttreatment assessment with one of the authors (P.F.S. or P.R.J.) who was blinded to treatment condition. This assessment consisted of the same structured interview for bulimic symptoms administered at pretreatment, the HDRS, GAFS, and EDI. In addition, the cue reactivity assessment was repeated at this time.

Summarizing the results of the trial, there were no significant differences across the three behavioral treatments on the percentage of individuals who were abstinent from binging or purging, or mean frequency of binging and purging at end-treatment. At the end of treatment, B-ERP, but not P-ERP, significantly reduced anxiety on the cue reactivity assessment, food restriction, body dissatisfaction, drive for thinness, and depression. These differences were not, however, maintained at the 6-month or 1-year follow-up evaluation. ${ }^{3}$

\section{One-Year Follow-up}

One year after the completion of treatment, all participants were contacted for a face-to-face follow-up evaluation. The subject and the assessor developed a lifechart for the 6 months before the evaluation. We enhanced the accuracy of recall by anchoring the 2-week epochs to memorable events in the patient's life and by including information that had been obtained previously in a face-to-face interview 6 months earlier and in a telephone interview 3 months earlier. The lifechart divided the 6-month interval into 2-week epochs, and the frequency of objective binging and purging during each epoch was recorded. We then calculated the mean frequency of binging and purging per 2-week period in the 3 months before the 1-year follow-up to develop an index of outcome based on the core behavioral features of BN. This definition reflected the diagnostic thresholds in DSM-IV (i.e., binging and compensatory behaviors occurred, on average, a minimum of twice per week for 3 months). For the 3 months before follow-up, the outcome categories were as follows: (1) no binging or purging; (2) some binging and/or purging, but both not more than twice per week on average; and (3) binging and purging twice per week or more on average.

\section{Subject Flow and Follow-up}

Of the 135 women who began the clinical trial, $106(78.5 \%)$ completed both the CBT and exposure/relaxation portions of the treatment. Of the 106 individuals who completed the treatment, 12-month follow-up data were available for 101 women ( $95 \%$ of completers and $75 \%$ of starters), who comprise the subjects of this report.

\section{Data Analyses}

Putative predictor variables were determined a priori during the development of the assessment batteries for this study and were assessed prospectively. Predictors were divided into three categories as follows: variables assessed at pretreatment about lifetime history (including personality), variables assessed at pretreatment about pretreatment status, and variables assessed at posttreatment about clinical status at the end of the treatment trial.

We first used univariate logistic regression ${ }^{11,12}$ to compute the measures of association between the putative predictors and the dependent variable (the trichotomous variable reflecting binging and total episodes of purging averaged over the 3 months before the 1-year follow-up evaluation). Next, we used stepwise logistic regression (setting the probability level to enter and leave the model at .15) within each of the three classes of predictors to determine the most parsimonious combination of predictors. Because these data were from a randomized clinical trial and the 1-year outcome could thus be influenced by the different treatment each individual received, variables coding the treatment received were stipulated to remain in all stepwise logistic regressions.

\section{RESULTS}

\section{Definition of Outcome}

Of the 101 women available for follow-up at 1 year, 17\% met DSM-III-R criteria for BN in the month before the 1-year follow-up assessment. Based on the frequency of binging and purging reported in the lifechart interviews in the 3 months before assessment, $38 \%$ of the sample reported no binging and purging, $45 \%$ reported some binging and/or purging but not more than twice per week on average, and $16 \%$ reported binging and purging twice per week or more on average. These $16 \%$ corresponded to the $17 \%$ of women who met DSM-III-R criteria at follow-up, with the slight difference attributable to missing data on one patient. The percentages of individuals in each outcome group who reported having received any additional treatment for their eating disorder in the interval between the end of treatment and the 1-year follow-up assessment were $2.6 \%$ (no binging or purging group), $6.7 \%$ (some binging and purging group), and $37.5 \%$ (twice per week or more group) $\left(\chi^{2}=12.1, P=.002\right)$.

\section{Treatment Group}

For each of the series of logistic regressions, treatment group was entered as a fixed predictor variable, meaning that these variables were constrained to remain in all regression models. In the lifetime and personality multivariate models, being randomized to the B-ERP treatment condition significantly decreased the odds of poor outcome at 1 year (odds ratio $[O R]=0.32 ; 95 \%$ confidence interval [CI], 0.12 to 0.91 ). Treatment group did not independently predict outcome in either the pretreat- 
ment or posttreatment multivariate regression analyses.

\section{Lifetime Variables Assessed at Pretreatment}

Table 1 presents univariate logistic regressions and stepwise multiple logistic regressions for the eight lifetime history, seven TCI, and three SCID-II variables assessed at pretreatment. None of the lifetime history variables significantly predicted outcome at 1 year in the univariate analyses. The TCI self-directedness scale significantly predicted outcome at 1 year with higher self-directedness associated with better outcome. For the cluster A (paranoid, schizoid, and schizotypal), cluster B (borderline, histrionic, narcissistic, and antisocial), and cluster $\mathrm{C}$ (obsessive-compulsive, dependent, avoidant, and passive-aggressive) symptom totals, none was significant in either the univariate or multivariate analyses. Given the interest in the literature in borderline personality disorder as a predictor of outcome, we conducted a univariate logistic regression using this personality disorder as the sole predictor. Borderline personality disor- der did not predict 1-year outcome $(\mathrm{OR}=1.29$ $95 \%$ CI, 0.55 to 3.04 ).

In the multivariate logistic regression, we entered all of the lifetime and personality variables as predictors. A lifetime history of obesity increased the odds of poor outcome by 7.9 ; a lifetime history of alcohol dependence decreased the odds of poor outcome by 0.26 ; and for each unit increase on the self-directedness scale, the odds of poor outcome were decreased by 0.94 .

\section{Pretreatment Status}

Table 2 presents the eight clinician-rated variables, three self-report variables, and three measures of cue reactivity that reflected clinical status at pretreatment. In the univariate analyses, lower GAFS, higher EDI bulimia scores, greater peak self-report urge to binge ratings on the cue reactivity assessment, and current major depression predicted poorer outcome at 1 year. In the multivariate analyses, GAFS, EDI bulimia, and major depression in the past month were retained in the stepwise procedure as significant independent predictors of

Table 1. Prediction of 1-Year Treatment Outcome of BN With Univariate and Stepwise Logistic Regression Using Variables With Demographics, Lifetime History, and Personality as Predictors

\begin{tabular}{|c|c|c|c|c|c|}
\hline \multirow[b]{2}{*}{ Lifetime Variables ${ }^{*}$} & \multirow{2}{*}{$\begin{array}{c}\text { Mean (SD) } \\
\quad \text { or } \%\end{array}$} & \multicolumn{2}{|c|}{ Univariate Logistic Regression } & \multicolumn{2}{|c|}{ Stepwise Logistic Regression } \\
\hline & & OR & $\mathrm{Cl}$ & OR & $\mathrm{Cl}$ \\
\hline Age (yr) & $26.5(6.13)$ & 0.97 & $0.91-1.03$ & $\ddagger$ & \\
\hline BMt minimum $\left(\mathrm{kg} / \mathrm{m}^{2}\right)$ & $18.6(2.46)$ & 0.95 & $0.81-1.10$ & $\ddagger$ & \\
\hline History of obesity (BMI >30) & $8.8 \%$ & 2.60 & $0.71-9.56$ & 7.86 & $1.42-43.64 t$ \\
\hline Prior inpatient treatment & $9.9 \%$ & 1.04 & $0.30-3.57$ & $\ddagger$ & \\
\hline Duration of BN (yr) & $6.82(6.07)$ & 0.96 & $0.91-1.03$ & $\ddagger$ & \\
\hline Lifetime anorexia nervosa & $24.3 \%$ & 1.09 & $0.46-2.60$ & $\neq$ & \\
\hline Lifetime major depression & $52.5 \%$ & 1.15 & $0.55-2.41$ & $\ddagger$ & \\
\hline Lifetime alcohol dependence & $42.6 \%$ & 0.81 & $0.38-1.72$ & $0.26 \dagger$ & $0.12-0.68 t$ \\
\hline Lifetime anxiety disorder $\S$ & $43.6 \%$ & 1.21 & $0.57-2.56$ & $\ddagger$ & \\
\hline Novelty seeking & $21.6(6.33)$ & 1.00 & $0.94-1.06$ & $\ddagger$ & \\
\hline Harm avoidance & $20.7(6.89)$ & 1.03 & $0.98-1.09$ & $\neq$ & \\
\hline Reward dependence & $15.8(4.36)$ & 1.03 & $0.95-1.12$ & $\ddagger$ & \\
\hline Persistence & $4.82(1.98)$ & 1.06 & $0.88-1.29$ & $\neq$ & \\
\hline Self-directedness & $24.5(8.20)$ & $0.94^{*}$ & $0.89-0.98 \dagger$ & $0.92 \dagger$ & $0.87-0.98 t$ \\
\hline Cooperativeness & $34.1(5.77)$ & 1.01 & $0.95-1.08$ & $\ddagger$ & \\
\hline Self-transcendence & $11.1(5.66)$ & 1.00 & $0.94-1.07$ & $\ddagger$ & \\
\hline Total cluster A symptoms & $4.12(3.45)$ & 1.02 & $0.91-1.14$ & $\neq$ & \\
\hline Total cluster B symptoms & $7.35(4.96)$ & 1.07 & $0.99-1.16$ & $\ddagger$ & \\
\hline Total cluster C symptoms & $6.36(4.64)$ & 1.02 & $0.94-1.10$ & $\ddagger$ & \\
\hline
\end{tabular}

Abbreviation: BMI, body mass index.

*Dependent variable: outcome 0 , no binging or purging; 1 , intermediate binging or purging; 2 , binging and purging $>2 \times /$ wk for 3 months. Treatment group fixed in all analyses. Receiving B-ERP was significantly and independently predictive of outcome at 1 year in these analyses.

tIndicates significance at the $\alpha<.05$ level.

\#ariable excluded in stepwise analysis.

§lncludes generalized anxiety disorder, social phobia, and obsessive-compulsive disorder; excludes simple phobia. 
Table 2. Prediction of 1-Year Treatment Outcome of BN With Univariate and Stepwise Logistic Regression Using Variables With Pretreatment Status as Predictors

\begin{tabular}{|c|c|c|c|c|c|}
\hline \multirow[b]{2}{*}{ Pretreatment Status } & \multirow[b]{2}{*}{ Mean (SD) or \% } & \multicolumn{2}{|c|}{ Univariate Logistic Regression } & \multicolumn{2}{|c|}{ Stepwise Logistic Regression } \\
\hline & & OR & $\mathrm{Cl}$ & OR & $\mathrm{Cl}$ \\
\hline Binges per 2-week period & $10.6(11.5)$ & 1.03 & $0.99-1.06$ & $\ddagger$ & \\
\hline Total purges per 2-week period & $14.7(20.8)$ & 1.03 & $1.00-1.06$ & $\ddagger$ & \\
\hline \multirow[t]{4}{*}{ Food restriction (quartiles) } & 3: $24 \%$ & & & & \\
\hline & $2: 29 \%$ & 129 & $088-188$ & \pm & \\
\hline & $1: 33 \%$ & $1 . \angle 8$ & $0.88-1.88$ & $\mp$ & \\
\hline & $0: 14 \%$ & & & & \\
\hline \multirow[t]{4}{*}{ Body dissatisfaction (quartiles) } & 3: $37 \%$ & & & & \\
\hline & 2: $35 \%$ & 097 & 064140 & 067 & $041-108$ \\
\hline & $1: 24 \%$ & 0.97 & $0.64-1.49$ & $0.6 /$ & $0.41-1.08$ \\
\hline & $0: \quad 4 \%$ & & & & \\
\hline GAFS & $55.6(6.66)$ & $0.91 t$ & $0.86-0.97 \dagger$ & $0.93 t$ & $0.86-0.99 \dagger$ \\
\hline HDRS & $8.75(5.39)$ & 1.07 & $0.99-1.15$ & $\ddagger$ & \\
\hline EDI: drive for thinness & $14.3(4.64)$ & 1.09 & $1.00-1.19$ & $\ddagger$ & \\
\hline EDI: bulimia & $9.61(4.78)$ & $1.15 t$ & $1.05-1.25 \dagger$ & $1.16 t$ & $1.06-1.27 \dagger$ \\
\hline EDI: body dissatisfaction & $18.9(7.50)$ & 1.03 & $0.98-1.08$ & $\ddagger$ & \\
\hline$\sqrt{\text { Peak SUDS }}$ & $1.67(0.83)$ & 1.45 & $0.68-3.12$ & $\ddagger$ & \\
\hline$\sqrt{\text { Peak urge to binge }}$ & $2.44(0.50)$ & 1.68 & $1.05-2.69$ & $\ddagger$ & \\
\hline$\sqrt{\text { Peak urge to purge }}$ & $2.04(0.95)$ & 1.34 & $0.89-1.98$ & $\ddagger$ & \\
\hline Major depression-past mo & $23 \%$ & $3.54 t$ & $1.39-9.01 \dagger$ & $2.80+$ & $1.04-7.52 \dagger$ \\
\hline Alcohol dependence--past mo & $16 \%$ & 1.16 & $0.42-3.18$ & $\neq$ & \\
\hline
\end{tabular}

*Dependent variable: outcome 0 , no binging or purging; 1 , intermediate binging or purging; 2 , binging and purging $>2 \times /$ wk for 3 Treatment months. Treatment group fixed in all analyses (see text for further details).

tIndicates significance at the $\alpha=.05$ level.

$\neq$ Variable excluded in stepwise analysis.

§Includes generalized anxiety disorder, social phobia, and obsessive-compulsive disorder; excludes simple phobia.

outcome. For each unit increase on the GAFS, the odds of good outcome increased by 0.93 . For each unit increase on the EDI bulimia scale, the odds of poor outcome increased by 1.16 . Finally, the presence of major depression at pretreatment increased the odds of poor outcome by 2.80 .

\section{Posttreatment Status}

In the univariate analyses, several of the posttreatment variables significantly predicted 1 -year outcome (Table 3). Poor outcome was predicted by the following: greater number of binges during the final 2-week period; greater food restriction; greater body dissatisfaction; lower GAFS; higher HDRS scores; higher EDI drive for thinness and bulimia scores; and higher peak SUDS, urge to binge, and urge to purge on the cue reactivity assessment. In the multivariate analyses, a greater number of binges per 2-week period, greater food restriction, and higher peak urge to binge on the cue reactivity assessment at the end of treatment independently predicted poorer outcome. In terms of magnitude of the effect, for each additional binge per 2-week period, the odds of poor outcome increased by 1.23 . For food restriction, our variable was coded in terms of quartiles; thus, for each increase in quartile that reflected an increase of intensity and/or frequency, the risk of poor outcome increased by a factor of 2.35 .

\section{DISCUSSION}

The primary aim of this study was to examine the prospective predictors of outcome 1 year after a clinical trial of CBT in 101 women with BN. We chose a definition of outcome anchored in the core behavioral features of the disorder (i.e., binging and purging), which reflected DSM-IV diagnostic thresholds and which was derived via a careful lifechart interview that reconstructed the individual's symptoms of BN in the 3-month interval before the 1-year follow-up assessment.

\section{The Effect of Treatment Received}

Given this was a randomized clinical trial, we fixed the variables that defined treatment group (i.e., B-ERP, P-ERP) to remain in the regression analyses. In the stepwise multivariate regression examining lifetime history variables, the odds of a favorable outcome at 1 year were increased if an individual had been assigned to B-ERP. B-ERP did 
Table 3. Prediction of One-Year Outcome of Bulimia Nervosa With Univariate and Stepwise Logistic Regression Using Variables Reflecting Posttreatment Status as Predictors

\begin{tabular}{|c|c|c|c|c|c|}
\hline \multirow[b]{2}{*}{ Posttreatment Variables* } & \multirow[b]{2}{*}{ Mean (SD) or \% } & \multicolumn{2}{|c|}{ Univariate Logistic Regression } & \multicolumn{2}{|c|}{ Stepwise Logistic Regression } \\
\hline & & OR & $\mathrm{Cl}$ & OR & $\mathrm{Cl}$ \\
\hline Binges per 2-week period & $1.58(3.24)$ & $1.30 \dagger$ & $1.11-1.51 \dagger$ & $1.23 t$ & $1.06-1.42 t$ \\
\hline Total purges per 2-week period & $3.67(8.03)$ & 1.10 & $1.03-1.18$ & $\ddagger$ & \\
\hline \multirow[t]{4}{*}{ Food restriction (quartiles) } & 3: $5 \%$ & & & & \\
\hline & 2: $15 \%$ & $2.45 t$ & $1.52-3.96 t$ & $2.35 t$ & $1.38-4.01 \dagger$ \\
\hline & 1: $34 \%$ & & & & \\
\hline & $0: 46 \%$ & & & & \\
\hline \multirow[t]{4}{*}{ Body dissatisfaction (quartiles) } & 3: $11 \%$ & & & & \\
\hline & 2: $24 \%$ & $3.25 \mathrm{t}$ & $189-5.58 t$ & $\neq$ & \\
\hline & 1: $52 \%$ & & & & \\
\hline & $0: 13 \%$ & & & & \\
\hline GAFS & $69.6(9.85)$ & $0.90 t$ & $0.86-0.95 \dagger$ & $\ddagger$ & \\
\hline HDRS & $5.15(5.64)$ & $1.11 \dagger$ & $1.04-1.20 t$ & $\ddagger$ & \\
\hline EDI: drive for thinness & $6.69(6.08)$ & $1.15 t$ & $1.07-1.24 \dagger$ & $\ddagger$ & \\
\hline EDI: bulimia & $2.23(3.26)$ & $1.23 \dagger$ & $1.09-1.40 t$ & $\ddagger$ & \\
\hline EDI: body dissatisfaction & $11.9(8.22)$ & 1.05 & $1.00-1.10$ & $\ddagger$ & \\
\hline$\sqrt{\text { Peak }}$ SUDS & $1.68(0.83)$ & $1.79 \dagger$ & $1.09-2.94 t$ & $\ddagger$ & \\
\hline$\sqrt{\text { Peak urge to binge }}$ & $0.79(0.92)$ & $2.11 t$ & $1.34-3.34 \dagger$ & $2.06 t$ & $1.24-3.43 \dagger$ \\
\hline$\sqrt{\text { Peak urge to purge }}$ & $0.80(0.98)$ & $2.81 \dagger$ & $1.76-4.47 \dagger$ & $\ddagger$ & \\
\hline
\end{tabular}

*Dependent variable: outcome 0 , no binging or purging; 1 , intermediate binging or purging; 2 , binging and purging $>2 \times / w k$ for 3 months. Treatment group fixed in all analyses.

tIndicates significance at the $\alpha=.05$ level.

$\neq$ Variable excluded in stepwise analysis.

not emerge as a significant predictor in either the pretreatment or posttreatment regressions, suggesting the magnitude of the effect is not sufficiently large when considering other more robust predictors. This finding reflects the results of the clinical trial wherein B-ERP was associated with positive outcome at the end of treatment with a fading effect at 6 months and 1 year.

\section{Lifetime History and Personality}

In terms of the lifetime history and personality predictors, a history of obesity increased the odds of poor outcome, and both a history of alcohol dependence and high self-directedness on the TCI decreased the odds of poor outcome. We did not find any predictive effect of duration of illness, unlike other studies that have found longer duration to be predictive of either poor outcome ${ }^{13-15}$ (suggesting that more entrenched behaviors are more difficult to change) or the opposite ${ }^{16}$ (suggesting that individuals who have suffered from the disorder for longer are more motivated to change). We were also unable to detect a curvilinear relationship between length of illness and outcome.

A history of obesity increased the odds of poor outcome by 7.9 (95\% CI, 1.42 to 43.6). Our findings are similar to those of Maddocks and
Kaplan, ${ }^{17}$ who found that poor responders tended to have a higher highest past weight than treatment responders. It is likely that having a history of obesity perpetuates dietary restriction, which in our subsequent analyses, was also predictive of poor outcome at 1 year.

Of our lifetime axis I comorbidity predictors, only alcohol dependence significantly predicted outcome. A lifetime diagnosis of anorexia nervosa, major depression, or any anxiety disorder (excluding simple phobia) did not significantly predict how an individual would fare at 1 year posttreatment. In contrast, a lifetime history of alcohol dependence actually decreased the odds of poor outcome. This finding is consistent with Strasser et al., ${ }^{18}$ who found that bulimic women with prior substance abuse had significantly lower posttreatment scores on self-reports of eating symptomatology, were more responsive to treatment, and displayed a better response to desipramine than bulimic women with no such history. Given that both of these studies examined past substance abuse or dependence, it is possible that success in overcoming one disorder is predictive of success in overcoming another.

The effect of personality disorders, especially borderline personality disorder, on treatment out- 
come in BN has been the subject of much debate. Several studies have found borderline personality disorder, borderline tendencies, or cluster B characteristics to be associated with poor outcome. ${ }^{15,19-22}$ This finding has not been universally replicated. ${ }^{23}$ In the present study, neither cluster B symptoms nor borderline personality disorder were significant predictors in either the univariate or multivariate analyses. In contrast, higher self-directedness, assessed by the TCI, was associated with superior outcome in both the univariate and multivariate regressions.

Cloninger et al. ${ }^{24}$ define self-directedness as a developmental process that encompasses several distinct aspects, including acceptance of responsibility for one's own choices, identification of individually valued goals and purposes, resourcefulness, and self-acceptance. Indeed, such characteristics appear to capture the optimal characteristics necessary for success in CBT. The presence of self-directedness at pretreatment may be a powerful predictor of suitability and treatment outcome in CBT. In a previous study, we found that selfdirectedness predicted the presence of personality disorder in women with BN. ${ }^{25}$ We argued that self-directedness, as measured by the TCI, may encapsulate succinctly a construct fundamental to the concept of "personality disorder." It may be this type of a unitary dimensional construct that predicts poor outcome rather than the presence or absence of more multifaceted construct of personality disorder. Self-directedness, but not personality disorder, has also been found to predict antidepressant response in individuals with major depression (P. Joyce, Personal Communication, August 1997). In light of these findings, the modifiability of the degree of self-directedness by the usefulness of techniques, such as motivational enhancement therapy ${ }^{26}$ or techniques to enhance readiness for change, ${ }^{27}$ remains an important empirical question.

\section{Pretreatment Clinical Status}

In the multivariate logistic regressions, lower global functioning, higher self-report bulimia scores on the EDI, and current major depression all increased the odds of having a poor outcome at 1 year. This finding is important for treatment planning. First, it appears that those individuals who, by the judgement of the clinician, are functioning more poorly across a host of domains, will do less well with CBT in the long run. Individuals with uncomplicated $\mathrm{BN}$, who are functioning well in both social and occupational domains, may fare better with a focused short-term treatment. For individuals with lower global functioning, it may be necessary to embed CBT for BN into a more comprehensive treatment package to address the breadth of clinical problems.

Second, unlike the actual frequency of binging and purging at pretreatment, severity of self-report bulimic symptoms and attitudes on the EDI was an independent predictor of outcome at 1 year. Whereas the actual frequency of binging and purging often waxes and wanes in women with $\mathrm{BN}$, the EDI self-report may be a better indicator of the more stable underlying construct of bulimic pathology. It could also reflect the degree to which an individual's bulimic symptoms are incorporated into her self-concept.

Finally, the odds of poor outcome were increased threefold in individuals who presented with major depression at pretreatment. This finding is consistent with several, ${ }^{17,28-30}$ but not all studies. ${ }^{23,31-33}$ One strength of our study is that depression at pretreatment was assessed with a structured diagnostic instrument rather than self-report scales. It is possible that the neurovegetative symptoms associated with depression interfere with engagement in the relatively focused and rapid-paced CBT in the clinical trial. Although not testable with this design, this finding could argue for the concurrent antidepressant treatment of individuals with $\mathrm{BN}$ who present with major depression to enhance utilization or efficacy of CBT. ${ }^{34}$

\section{Posttreatment Clinical Status}

The last series of analyses addressed whether there are features of the clinical status at the end of a therapeutic trial of CBT that can predict outcome at 1 year. This question is informative in several ways. First, it can highlight important symptoms to target during treatment. For example, in our study, the presence of binging behavior at the end of treatment presaged poor outcome. For each additional binge per 2-week period, the odds of poor outcome at 1 year increased by 1.23 . These findings support the assertion of Maddocks et al. ${ }^{35}$ that abstinence is a critical dimension of outcome.

Similarly, if an individual continued to have a restrictive pattern of eating at the end of treatment, or if she continued to have a strong urge to binge in response to high-risk foods, her odds of poor 
outcome were similarly increased. Thus, abstinence from binging, elimination of restrictive eating patterns (i.e., dieting), and decreases in cue reactivity (especially urge to binge) are important therapeutic goals to achieve before termination of treatment.

These results must be interpreted with several caveats in mind. First, the validity of any regression analysis hinges on the choice of predictors. Although we attempted to be parsimonious in our choices, this still resulted in a relatively large number of putative predictor variables. Variables that we did not include could have had superior predictive capacity. Second, our results are specific for CBT, and the same set of predictors may not significantly predict outcome for other types of psychotherapy or for pharmacological treatment. Third, by choice, these analyses focused only on those individuals who engaged in treatment and who were available for follow-up. These predictors have no bearing on determining who is likely to remain in treatment versus discontinuing prematurely.

In summary, the presence of impaired global functioning, high self-report bulimia on the EDI, and current major depression should alert clinicians that a narrow focused approach to the treatment of BN may not be sufficient to address the range of clinical problems, and that more broad-based, possibly multimodal treatment may be optimal for these individuals to ensure long-term positive outcome. Second, individuals with a history of obesity may be at risk for poor outcome, whereas a history of alcohol dependence may portend success. Third, the personal quality of self-directedness may be a strong indicator of readiness and ability to change at pretreatment. Approaches to enhance selfdirectedness may assist with augmenting the effect of CBT for individuals who are low on this dimension. Finally, the results of this study support the therapeutic goal of abstinence from binging and dieting behavior and a reduction in the urge to binge in response to high risk cues, as the presence of these features at the end of treatment is associated with poor long-term outcome. Treatment that is terminated before these behaviors have been controlled may compromise long-term recovery.

\section{REFERENCES}

1. Keel P, Mitchell J. Outcome in bulimia nervosa. Am J Psychiatry 1997;154:313-321.

2. Keller M, Herzog D, Lavori P, Bradburn I, Mahoney E. The naturalistic history of bulimia nervosa: Extraordinarily high rates of chronicity, relapse, recurrence, and psychosocial morbidity. Int J Eat Disord 1992;12:1-9.

3. Bulik C, Sullivan P, Carter F, Joyce P. The role of exposure with response prevention in the cognitive-behavioral therapy for bulimia nervosa. Psychol Med. In press.

4. Spitzer R, Williams J, Gibbon M, First M. Structured Clinical Interview for DSM-III-R: Patient Version (SCID-P, 4/1/88). New York, NY: Biometrics Research Department, New York State Psychiatric Institute, 1988.

5. Spitzer R, Williams J, Gibbon M. Structured Clinical Interview for DSM-III-R: Personality Disorders (SCID-II, 3/1/ 87). New York, NY: Biometrics Research Department, New York State Psychiatric Institute, 1987.

6. Fairburn C, Cooper Z. The Eating Disorders Examination. Ed. 12. In: Fairburn C, Wilson G (eds): Binge-Eating: Nature, Assessment and Treatment. New York, NY: Guilford, 1993:317360 .

7. Beglin S, Fairburn C. What is meant by the term "binge"? Am J Psychiatry 1992;149:123-124.

8. Hamilton M. A rating scale for measuring depression. J Neurol Neurosurg Psychiatry 1960;23:56-62.

9. Garner D, Olmsted M, Polivy J. Eating Disorders Inventory Manual. New York, NY: Psychological Assessment Resources, 1984.

10. Cloninger C, Przybeck T, Svrakic D, Wetzel R. The Temperament and Character Inventory (TCI): A Guide to its
Development and Use. St. Louis, MO: Center for Psychobiology of Personality, Washington University, 1994.

11. SAS Institute Inc. SAS/STAT = AE User's Guide version 6. Cary, NC: SAS Institute, 1989.

12. SAS Institute Inc. SAS/STAT $=$ AE Software: Changes and Enhancements. Cary, NC: SAS Institute, 1996.

13. Herzog D, Sacks N. Bulimia nervosa: Comparison of treatment responders vs. nonresponders. Psychopharmacol Bull 1993;29:121-125.

14. Hsu LKG, Holder D. Bulimia nervosa: Treatment and short-term outcome. Psychol Med 1986;16:65-70.

15. Fahy T, Russell G. Outcome and prognostic variables in bulimia nervosa. Int J Eat Disord 1993;12:135-145.

16. Turnbull S, Schmidt U, Troop N, Tiller J, Todd G, Treasure J. Predictors of outcome for two treatments for bulimia nervosa: Short and long term. Int J Eat Disord 1997;21:17-22.

17. Maddocks SE, Kaplan AS. The prediction of treatment response in bulimia nervosa. Br J Psychiatry 1991;159:846-849.

18. Strasser T, Pike K, Walsh B. The impact of prior substance abuse on treatment outcome for bulimia nervosa. Addict Behav 1992;17:387-395.

19. Herzog T, Hartmann A, Sandholz A, Stammer H. Prognostic factors in outpatient psychotherapy of bulimia. Psychother Psychosom 1991;56:48-55.

20. Rossiter E, Agras W, Telch C, Schneider J. Cluster B personality disorder characteristics predict outcome in the treatment of bulimia nervosa. Int J Eat Disord 1993:13:349-357.

21. Johnson C, Tobin D, Dennis A. Differences in treatment outcome between borderline and nonborderline bulimics at one-year follow-up. Int J Eat Disord 1990;9:617-628. 
22. Steiger H, Stotland S, Houle L. Prognostic implications of stable versus transient "borderline features" in bulimic patients. J Clin Psychiatry 1994;55:206-214.

23. Olmsted M, Kaplan A, Rockert W. Rate and prediction of relapse in bulimia nervosa. Am J Psychiatry 1994;151:738-743.

24. Cloninger CR, Svrakic DM, Przybeck TR. A psychobiological model of temperament and character. Arch Gen Psychiatry 1993;50:975-990.

25. Bulik C, Sullivan P, Carter F, Joyce P. Temperament, character, and personality disorder in bulimia nervosa. J Nerv Ment Dis 1995;183:593-598.

26. Treasure J, Ward A. A practical guide to the use of motivational interviewing in anorexia nervosa. Eur Eat Disord Rev 1997;5:102-114.

27. Prochaska J, Norcross J, DiClimente C. Changing for Good. New York, NY: Avon, 1994.

28. Bossert S, Schmolz U, Wiegand M, Junker M, Kreig J-C. Case history and shorter communication: Predictors of shortterm treatment outcome in bulimia nervosa patients. Behav Res Ther 1992;30:193-199.

29. Brotman A, Herzog D, Hamburg P. Long-term course in
14 bulimic patients treated with psychotherapy. J Clin Psychiatry 1988;49:157-160.

30. Fichter M, Quadflieg N, Rief W. Longer-term course (6-year) course of bulimia nervosa. Neuropsychopharmacology 1994;10:772S.

31. Herzog DB, Keller MB, Lavori PW, Sacks NR. The course and outcome of bulimia nervosa. J Clin Psychiatry 1991;52(Suppl):4-8.

32. Walsh B, Hadigan C, Devlin M, Gladis M, Roose S. Long-term outcome for antidepressant treatment for bulimia nervosa. Am J Psychiatry 1991;148:1206-1212.

33. Mitchell J, LD, Goff G, Pyle R. A follow-up study of patients with bulimia. Int J Eat Disord 1986;5:441-450.

34. Walsh B, Wilson G, Loeb K, Devlin M, Pike K, Roose S, et al. Medication and psychotherapy in the treatment of bulimia nervosa. Am J Psychiatry 1997;154:523-531.

35. Maddocks SE, Kaplan AS, Woodside DB, Langdon L, Piran N. Two year follow-up of bulimia nervosa: The importance of abstinence as the criterion outcome. Int $\mathrm{J}$ Eat Disord 1992;12:133-141. 